potentially a lot more to the community than accepted under the mental illness umbrella.

I raise this issue again with the hope of extending our roles not only to the 'Holy Grail' of reducing costs and improving outcomes, as the editorial focuses, but also to the wider losses our community and society suffer but are unable to react to. On another note, the editorial mentions the RAID model (Rapid Assessment Interface and Discharge). This along with the latest iteration of the National Institute for Health and Care Excellence guidance on schizophrenia, ${ }^{4}$ which refocuses attention on combined physical and mental healthcare and the mandate around parity with physical and mental healthcare just debated in the English Parliament, ${ }^{5}$ gives us hope for the future. Psychiatrists are unique in addressing the boundary disputes between specialties and offer value for money even in this economy.

1 Sharpe M, Psychological medicine and the future of psychiatry. Br J Psychiatry 2014; 204: 91-2.

2 Appleby L, Kapur N, Shaw J, Hunt IM, While D, Flynn S, et al. The National Confidential Inquiry into Suicide and Homicide by People with Mental IIIness. Annual Report: England, Northern Ireland, Scotland and Wales. The University of Manchester, 2013.

3 World Health Organization. The ICD-10: Classification of Mental and Behavioural Disorders: Clinical Description and Diagnostic Guidelines. WHO, 1992.

4 National Institute for Health and Care Excellence. Psychosis and Schizophrenia in Adults: Treatment and Management (NICE Clinical Guideline 178). NICE, 2014.

5 NHS England. NHS England welcomes refreshed Mandate from the Government. NHS England, 2013; 12 November (http://www.england. nhs.uk/2013/11/12/mandate-response/).

Mukesh Kripalani, Tees, Esk and Wear Valleys NHS Foundation Trust, UK. Email: drmukesh@doctors.org.uk

doi: 10.1192/bjp.204.6.492a

Author's reply: It is encouraging that the ideas expressed in my editorial on psychological medicine have stimulated such interest and associated correspondence. The three letters published above support the thrust of the editorial that a re-engagement of psychiatry with other areas of medicine in the form of psychological medicine services (also called liaison psychiatry) would benefit both medicine and psychiatry. Each letter also raises specific additional points.

Rowett \& Udo doubt whether psychiatry is up to the 'sheer scale of the task' in helping other areas of medicine to address the 'compassion vacuum' highlighted by the Francis Inquiry. They conclude that medicine should put its own house in order by re-engaging with its patients and carers rather than seek solutions from another specialty. They are clearly correct in noting that the task is great and that the change required cannot be delivered by psychiatry alone. But I think they are too pessimistic, both about the appetite for change within medicine and about how much can be achieved by psychological medicine; it cannot transform medicine on its own, but it can be an important facilitator of change.

Mukaetova-Ladinska \& Scully emphasise the importance of old age psychiatry in light of the rising age of general medical patients. They argue for the specific development of liaison psychiatry of old age. Although fully agreeing with them that expertise in the psychiatry of old age is an essential ingredient of a modern psychological medicine service, I am less convinced of the merit of subspecialised services. Integration with medical services requires that we map onto the way in which they are provided and the very demographic trend they have highlighted is breaking down the division between adult medicine and geriatrics. Hence although the skills of old age psychiatry are increasingly important for psychological medicine services, setting up service barriers defined by age is unlikely to achieve effective integration with medicine.

Finally, Kripalani makes the important point that we need to consider the role of psychiatry in ensuring patient safety. The point is made that services which concentrate on 'severe mental illness' may miss the risk of suicide posed by the individual suffering from stress and adjustment disorders. I am sure that most practitioners working in psychological medicine services would endorse this point. Psychological medicine can play an important role in helping medical services to reduce risk, as well as in improving patient outcomes and experience and making medical care more efficient.

I wish to thank these correspondents, and others who have emailed me personally, for their interest in the points raised in the editorial. The opportunities for psychiatry to re-engage with clinical medicine are enormous. I would strongly urge all those with an interest in developing integrated patient-centred psychological medicine services to help psychiatry to rise to this challenge. Our patients and our specialty need us to succeed.

Michael Sharpe, University of Oxford Department of Psychiatry, Warneford Hospital Oxford OX3 7JX, UK. Email: michael.sharpe@psych.ox.ac.uk

doi: 10.1192/bjp.204.6.493

\section{Assessing and staging bipolar disorder}

We congratulate Duffy et al on their paper. ${ }^{1}$ We have long argued that bipolar disorder is often underdiagnosed by community mental health teams, and that the reason for this is often failure to assess the longitudinal trajectory of patients with recurrent depression. ${ }^{2,3}$ We have attempted to remedy this by developing 29 questions to be used in the history-taking of all patients with depression and recurrent depression to demonstrate the developmental trajectory of the illness. ${ }^{4}$ These questions are presently being field tested in Bedford, UK, and at the University of Perugia, Italy. We have also demonstrated that when the systematic assessment of the trajectory of bipolar disorder is carried out in a community mental health team, the number of patients with bipolar disorder among the patients assessed by the team increases, but there remain a number of patients who do have unipolar depression; ${ }^{5}$ in other words, the assessment of the trajectory of patients with mood disorder enables the discrimination between bipolar and unipolar depression.

We would comment that Duffy et al raise an important point in suggesting that a history of use of lithium by relatives of the patients changes the trajectory of bipolar disorder; however, in our experience it is very difficult to collect this information from patients, who often do not know details of their relatives' illnesses. Furthermore, Duffy et al are right in proposing that it is possible to suggest a staging of bipolar disorder similar to McGorry's staging of schizophrenia, but the schizophrenia staging is underpinned by Pantelis' neuroimaging of the different stages of schizophrenia. To propose a staging model of bipolar disorder, we require similar neuroimaging results describing the differences between the individual stages.

1 Duffy A, Horrocks J, Doucette S, Keown-Stoneman C, McCloskey S, Grof P. The developmental trajectory of bipolar disorder. Br J Psychiatry 2014; 204: $122-8$.

2 Rogers J, Agius M. Bipolar and unipolar depression. Psychiatr Danub 2012; 24 (suppl 1): 100-5 
3 Rogers J, Agius M, Zaman R. Diagnosis of mental illness in primary and secondary care with a focus on bipolar disorder. Psychiatr Danub 2012; 24 (suppl 1): 86-90.

4 Agius M, Murphy $\mathrm{H}$. Proving that a patient has bipolar disorder. Cut Edge Psychiatry Pract 2013; 1: 174-80.

5 Bongards EN, Zaman R, Agius M. Can we prevent under-diagnosis and misdiagnosis of bipolar affective disorder? Repeat audits to assess the epidemiological change in the caseload of a community mental health team when bipolar disorder is accurately assessed and diagnosed. Psychiatr Danub 2013; 25 (suppl 2): 129-34.

Mark Agius, Clare College, Cambridge, UK. Email: ma393@cam.ac.uk; Jonathan Rogers, Caius-Gonville College, Cambridge, UK; Eva Bongards, Christ's College, Cambridge, UK; Stuart O'Connor, University of Warwick, UK; Norma Verdolini, University of Perugia, Italy; Sandro Elisei, University of Perugia, Italy

doi: 10.1192/bjp.204.6.493a

Author's reply: In the article ${ }^{1}$ we presented data describing the clinical stages in developing bipolar disorder based on longitudinal repeated assessment of the offspring of well-characterised parents with bipolar disorder. The findings emphasise the importance of including family history and clinical course in the diagnostic formulation to improve early identification, given that early risk syndromes are non-specific and include anxiety and depressive syndromes. We also showed that offspring of lithium-responsive parents develop classic episodic mood disorders, whereas offspring of parents with a lithium non-responsive illness follow a trajectory that overlaps with psychotic disorders - both in early and end-stage disorders. We did not suggest that 'a history of lithium use in relatives changes the trajectory', rather we used an operationalised published protocol to identify a more homogeneous subtype of bipolar disorder based on the excellent response to long-term lithium in the affected parent. ${ }^{2}$ Second, the staging model proposed by McGorry and colleagues ${ }^{3}$ was originally based on clinical observations in help-seeking youth (clinically high risk) and only later validated by conversion rates to psychosis and more recently neurobiological findings. Our cohort is a genetically high-risk cohort and we are intensively investigating markers of illness predisposition and progression through the clinical staging model. ${ }^{4}$ In regard specifically to neuroanatomical markers, in collaboration we have reported that enlarged right inferior frontal gyrus volumes may be a marker of bipolar disorder predisposition in high-risk offspring. ${ }^{5}$

1 Duffy A, Horrocks J, Doucette S, Keown-Stoneman C, McCloskey S, Grof P. The developmental trajectory of bipolar disorder. Br J Psychiatry 2014; 204: $122-8$.

2 Grof $\mathrm{P}$, Duffy A, Alda $\mathrm{M}$, Hajek $\mathrm{T}$. Lithium response across generations. Acta Psychiatr Scand 2009; 120: 378-85.

3 McGorry PD, Nelson B, Goldstone S, Yung AR. Clinical staging: a heuristic and practical strategy for new research and better health and social outcomes for psychotic and related mood disorders. Can J Psychiatry 2010; 55: 486-97.

4 Duffy A, Horrocks J, Doucette S, Keown-Stoneman C, Grof P, Andreazza A, et al. Immunological and neurotrophic markers of risk status and illness development in high-risk youth: understanding the neurobiological underpinnings of bipolar disorder. Int J Bipolar Disord 2014; 2: 4.

5 Hajek T, Cullis J, Novak T, Kopecek M, Blagdon R, Propper L, et al. Brain structural signature of familial predisposition for bipolar disorder: replicable evidence for involvement of the right inferior frontal gyrus. Biol Psychiatry 2013; 73: 144-52.

Anne Duffy, Mathison Centre for Mental Health Research, 4th Floor TRW Building, Room 4D68, Calgary, Alberta T2N 4Z6, Canada. Email: acduffy@ucalgary.ca

doi: 10.1192/bjp.204.6.494

\section{Correction}

Stimulant treatment for attention-deficit hyperactivity disorder and risk of developing substance use disorder. BJP, 203, 112-119. In Table 1 (p. 115): of those with ADHD in the no-stimulant treatment group, the percentage of males is $59 \%$.

Anyone can experience a major traumatic event; some are more likely to than others but most of us will suffer trauma at some time in our lives. Most traumatic experiences are processed through a normal response, with or without distress but without the development of mental disorder. Traumatic memories characteristic of post-traumatic stress disorder are unbidden, intrusive, vivid, distressing and accompanied by avoidance of them and their triggers. They are often amenable to treatment; trauma-focused psychological therapies are the treatments of choice. Some medications and non-trauma-focused psychological therapies can reduce the intensity of traumatic memories and their impact on functioning. 